\title{
THE IMPACT OF THE FLC CONTROLLER'S SETTINGS ON THE PRECISION OF THE POSITIONING OF A PAYLOAD TRANSFERRED BY A MOBILE CRANE
}

\author{
Jacek KŁOSIŃSKI*, Jarosław JANUSZ*, Rafał NYCZ* \\ "Faculty of Mechanical Engineering and Computer Science, University of Bielsko-Biała, ul. Willowa 2, 43-309, Bielsko-Biała, Poland \\ jklosinski@ath.bielsko.pl, jianusz@ath.bielsko.pl, rafal.nycz@op.pl
}

\begin{abstract}
The paper presents a model of a control system of the slewing motion of a mobile crane in which the FLC controller was used, and then selected results of the numerical simulations of this model were presented. The influence of this controller's settings on the precision with which the payload is positioned after it has been transferred to a target point for different angles of rotation of the jib, different lengths of the rope and different input signals of the controller was investigated.
\end{abstract}

Key words: Mobile Crane, FLC Controller

\section{INTRODUCTION}

The oscillations of the payload that is transferred by lifting equipment considerably hinder the work of the device and increase transportation time, especially because of the time that is needed to damp these oscillations, and they may also constitute a threat to the operators and technological facilities that are located in the vicinity of the working equipment (Al-Humaidi and Hadipriono, 2009; Janusz and Kłosiński, 2008; Kłosiński and Majewski, 2009; Kłosiński, 2011). They may also hinder the positioning of the payload after it has been transferred to a target point - this is particularly important when heavy payloads are moved (Cho and Lee, 2002; Neupert et al., 2010; Yi et al., 2003). Therefore, in certain lifting devices control systems are installed. Such systems make it possible to minimise the oscillations of the payload at a target point. These are usually systems that implement a particular strategy of controlling the working motion which ensures that the carried payload stops at a target point and that, at the same time, the payload's oscillations are eliminated. When disturbances appear or the implementation of the adopted control strategy is interrupted, these systems do not ensure minimising the oscillations of the payload at a target point (Lee and Lee, 2002; Tomczyk et al., 2014). The transfer of the payload by using the rotational motion of a lifting device, as is the case with slewing, mobile or tower cranes, causes particular difficulty in this regard. Under these conditions, the use of a fuzzy logic controller seems to be a solution that may ensure higher quality control than classical control methods (Cho and Lee, 2002; Liu et al., 2005; Smoczek, 2014; Smoczek et al., 2013; Smoczek and Szpytko, 2013; Solihin et al., 2010).

This paper presents a model of a control system of the slewing motion of a mobile crane in which the FLC controller was used, and then selected results of the numerical simulations of this model were presented. The influence of this controller's settings on the precision with which the payload is positioned after it has been transferred to a target point for different angles of rotation of the jib, different lengths of the rope and different input signals of the controller was investigated.

\section{MODEL OF A CONTROL SYSTEM OF THE WORKING MOTIONS OF A MOBILE CRANE}

The analyses of models which are presented in other papers allow one to make assumptions with regard to creating a model of a crane (Hong and Ngo, 2012; Jerman et al., 2004; Ju et al., 2006; Schaub, 2008; Sochacki, 2007; Tereshima et al., 2007). This model is aimed to investigate the slewing motion of a crane's body. The following assumptions have been made (Kłosiński, 2005; Kłosiński and Janusz, 2009):

- the solids of the chassis and the body, of known masses and moments of inertia, are rigid and have six degrees of freedom; the only possible motion of the body in relation to the chassis is its rotation around the vertical axis;

- the support system was substituted with a set of springs; the mass of springs is omitted;

- the crane's body is rotated by means of a hydrostatic drive system through a mechanical gear of known rigidity, the centre of mass of the body lies on the axis of rotation of the body;

- the crane's jib is treated as a rigid bar of constant length, of known mass and moment of inertia; it is connected with the body by means of a cylindrical joint which only allows for a change in the jib's inclination angle;

- the payload hangs on an inextensible, weightless, flexible rope; the hanging payload can be treated as a spherical pendulum;

- the drum of the winch is rotated by means of a hydrostatic drive system through a mechanical gear of known rigidity,

- friction and clearances in all elements of the support system, jib and mechanical gear are not taken into consideration;

- damping in the system is taken into consideration;

- the characteristics of elastic constraints are assumed to be linear,

- three working motions are possible: the slewing motion of the crane's body, luffing as a consequence of a change in the jib's inclination angle which is caused by a change in the length of the hydraulic cylinder as well as the hoisting or lowering 
of the payload as a consequence of reeling or unreeling the rope; a simultaneous association of all these motions is permitted;

- all supports of the crane are treated as unilateral constraints, the supports can come off the foundation.

A schematic diagram of the crane's model is presented in Fig. 1.

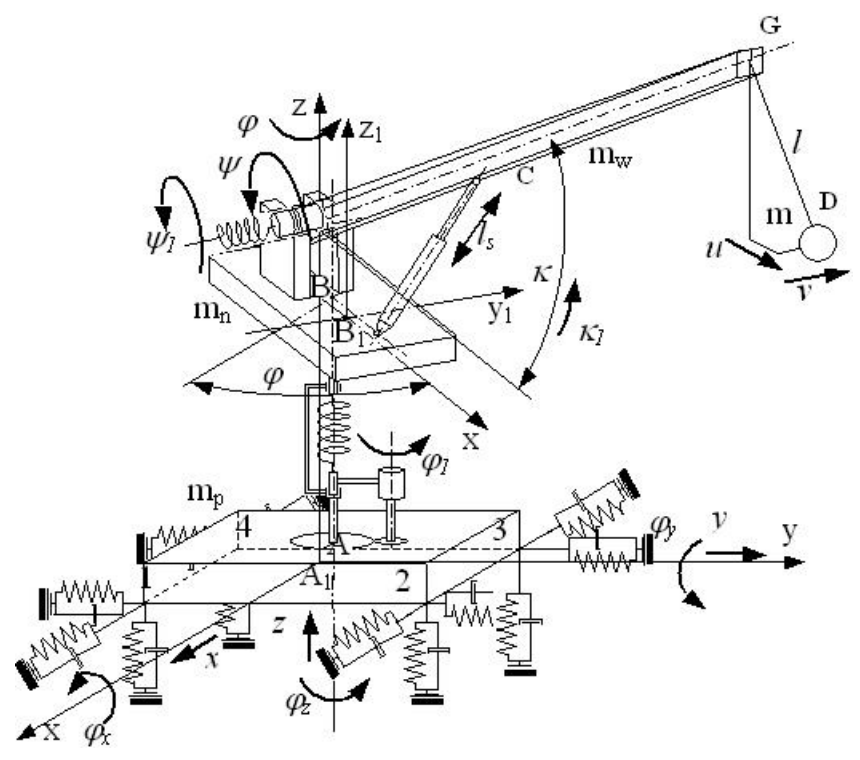

Fig. 1. Schematic diagram of crane model (Kłosiński, 2005)

Generalized co-ordinates of the model are put together in the n-element vector $q$. Its co-ordinates are as follows:

$q=\left[\begin{array}{lllllllll}x & y & z & u & v & \varphi_{x} & \varphi_{y} \varphi_{z} \kappa & l\end{array}\right]^{T}$

where: $x, y, z$ - linear displacements of the centre of mass of the chassis which is referred to as a fixed reference system, $u, v$ - projections of the payload's displacement onto a horizontal plane in the radial direction, and in the tangential direction to the circle described by jib's head, respectively; these components are determined in a movable system rotating with the jib, $\varphi$ - angle of rotation of the body and the jib round a vertical axis, $\varphi_{x}, \varphi_{y}, \varphi_{z}$ - angles of oscillation of the crane in reference to the axes that are connected with the centre of mass of the chassis, $\kappa$ - the jib's inclination angle in reference to the horizontal plane, $l$ - distance from the jib's head to the centre of gravity of the payload.

When calculating the kinetic and potential energies of the system and inserting the obtained formulas into the second-order Lagrange equation, which is written in the form:

$\frac{\mathrm{d}}{\mathrm{d} t}\left(\frac{\partial E_{k}}{\partial \dot{q}}\right)-\frac{\partial E_{k}}{\partial q}+\frac{\partial E_{p}}{\partial q}+\frac{\partial E_{R}}{\partial q}=0$

one can write a matrix, non-linear, differential equation in the following form (Kłosiński, 2005):

$M(q) \ddot{q}=P(\dot{q}, \ddot{q})$

where: $M(q)$ - symmetrical block matrix whose elements are functions of masses, the inertial moments of the model's elements and generalized co-ordinates, $P(\dot{q}, \ddot{q})$ - n-element vector whose components are functions of generalized coordinates and their derivatives.
This equation can be transformed into an equation in the following form:

$\ddot{q}=f(\dot{q}, q)+b(q) q_{u}$

where: $q_{u}=\left[\begin{array}{lll}\varphi_{1} & \kappa_{1} & \psi_{1}\end{array}\right]^{T}, \varphi_{1}$ - rotational angle of the shaft of the hydraulic motor which is used for putting the crane's body with the jib in slewing motion, $\kappa_{1}$ - inclination angle of the jib's longitudinal axis which is related to the horizontal plane; the axis is determined by two points: the point of vertical rotation of the jib and the point of fixing of the luffing cylinder to the jib, $\Psi_{1}$ - rotational angle of the motor-driven drum of the winding machine, $b(q)$ - vector whose components are functions of generalized coordinates and stiffness coefficients of elastic elements.

Formula (4) represents a motion equation of the crane model in matrix form describing the slewing motion of its body, changes in the jib reach through changes in the declination of the jib as well as the hoisting or lowering of the payload through winding or unwinding the rope from the drum. The components of the oscillations of the payload as well as the angle of rotation of the body can be calculated by means of the following equations:

$v=c_{1}^{T} q \quad u=c_{2}^{T} q \quad \varphi=c_{3}^{T} q$

where:

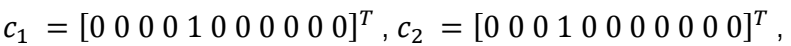
$c_{3}=\left[\begin{array}{lllllllllll}0 & 0 & 0 & 0 & 0 & 1 & 0 & 0 & 0 & 0 & 0\end{array}\right]^{T}$.

The mathematical models of drive systems that are used for different work motions were derived in a similar way. They were proposed in the form of hydrostatic systems powered by engines with constant absorbing capacity (or by a servo-motor) and proportional valves as the elements which throttle oil flow (Kłosiński, 2005; Kłosiński and Janusz, 2009).

Apart from the equation of these models, i.e. equation (4), and formulas modelling the dynamics of fuzzy logic controller and the equation of the summation point, equations were obtained which were considered a mathematical model of the complete control system. A functional scheme of the control system is presented in Fig. 2 (Janusz and Kłosiński, 2008). In the scheme, the character e was used to describe an error signal which is an input to the controller. The character $i$ describes a vector of variables which control the work of the hydrostatic systems that are used as the drive of a crane; these are usually currents of electro-hydraulic transformers that control hydraulic drive systems which are used in cranes.

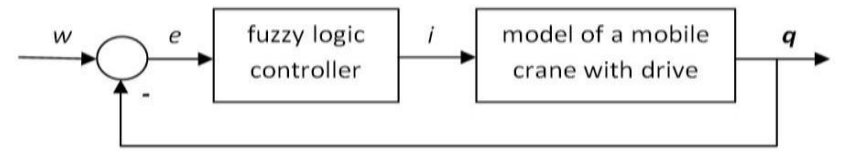

Fig. 2. Functional scheme of control system of mobile crane

\section{SIMULATION INVESTIGATIONS OF THE SYSTEM}

Numerical investigations of the model were performed for a Mamdani-type fuzzy logic controller (Kłosiński and Majewski, 2004; Kłosiński, 2011; Lee and Lee, 2002; Mahfouf et al., 2000; Pędrak and Kłosińki, 2009) which was used as a controller in the system. For slewing motion the following main elements of the controller can be distinguished:

- fuzzification block; input signals used in the simulation investigations were as follows: 
- tangent co-ordinate of payload swings $v$ and the angle distance of the jib from a target point, i.e.: $\Delta \varphi=\varphi_{z a d}-\varphi$ or angle distance of the jib from a target point $\Delta \varphi=\varphi_{z a d}$ $\varphi$ and angular velocity;

- fuzzification was performed by using triangular membership functions;

- qualifier block - based on the assumed rule database; a database consisting of 25 reasoning rules was used in the performed numerical simulations (Mahfouf et al., 2000; Pędrak and Kłosiński, 2009),

- defuzzification block - based on the centre-of-gravity method - cos.

Calculations were carried out for the above-mentioned combinations of input signals of the rotation motion controller, different lengths of the rope on which the payload was suspended and different angles of rotation of the jib as well as different values of the coefficients of amplification of particular signals in the controller. The amplification coefficients refer to the input and output signals of the controller. Selected results are presented in the form of graphs in Figs. 3-6.

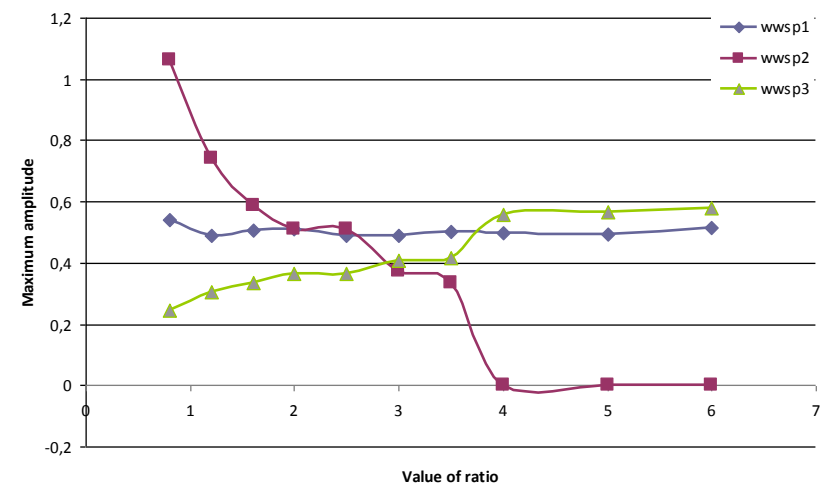

Fig. 3. Diagrams: max amplitude of the payload swings remained after ending of the rotating movement vs amplification coefficient of the controller input (wwsp1, wwsp2) and output (wwsp3) signals $\left(\varphi_{\text {end }}=1.57 \mathrm{rad}, l=4 \mathrm{~m}\right)$

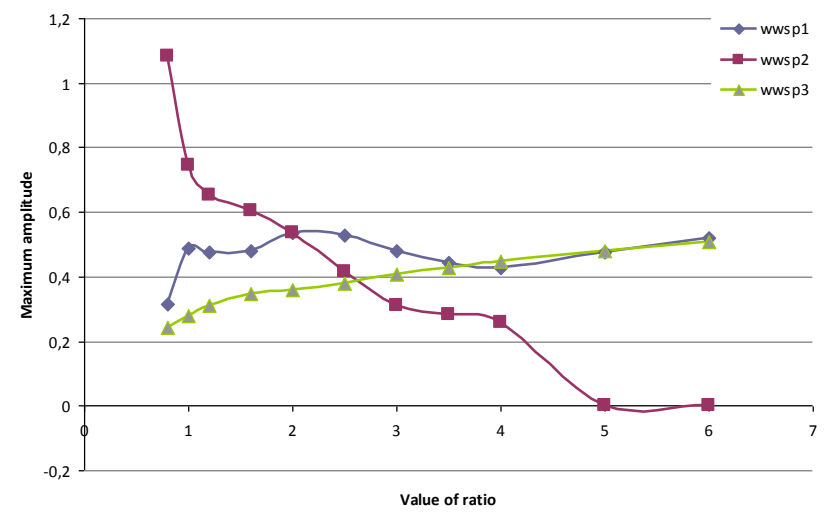

Fig. 4. Diagrams: max amplitude of the payload swings remained after ending of the rotating movement vs amplification coefficient of the controller input (wwsp1, wwsp2) and output (wwsp3) signals $\left(\varphi_{\text {end }}=1.05 \mathrm{rad}, l=4 \mathrm{~m}\right)$

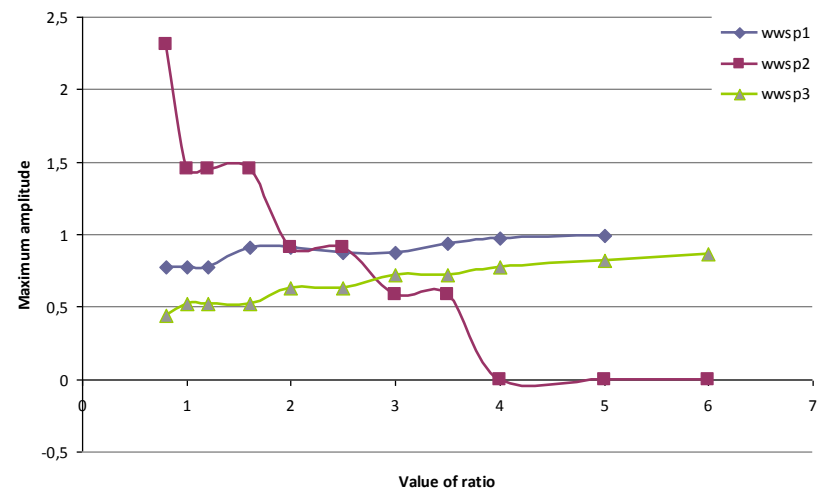

Fig. 5. Diagrams: max amplitude of the payload swings remained after ending of the rotating movement vs amplification coefficient of the controller input (wwsp1, wwsp2) and output (wwsp3) signals $\left(\varphi_{\text {end }}=1.57 \mathrm{rad}, l=12 \mathrm{~m}\right)$

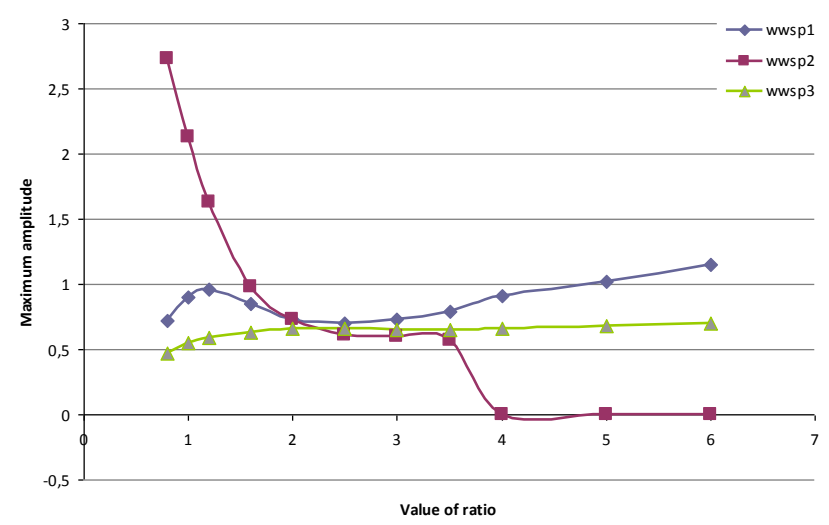

Fig. 6. Diagrams: max amplitude of the payload swings remained after ending of the rotating movement vs amplification coefficient of the controller input (wwsp1, wwsp2) and output (wwsp3) signals $\left(\varphi_{\text {end }}=1.05 \mathrm{rad}, l=12 \mathrm{~m}\right)$

The graphs showing the dependence of the maximum amplitude of the oscillations of the payload after a rotation and after the jib stops at a target point as a function of signal amplification coefficients in the FLC controller allow one to adopt a satisfactory value of the controller's settings.

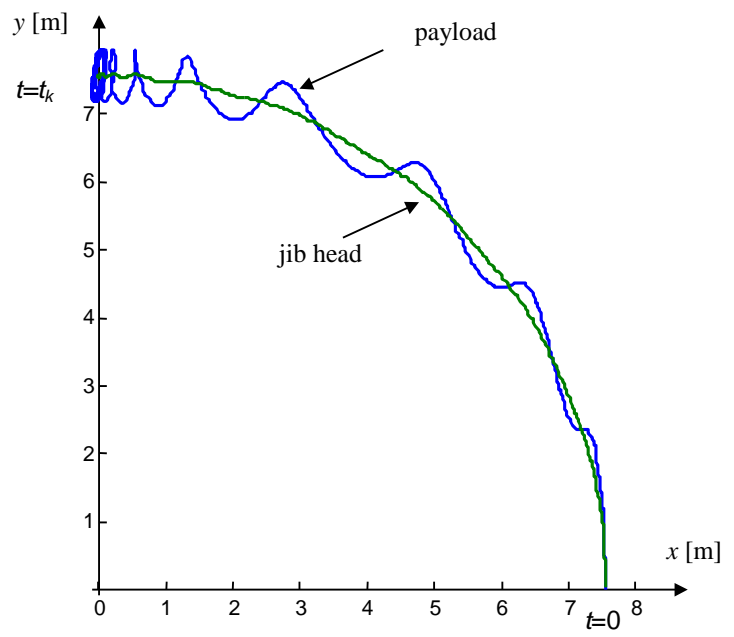

Fig. 7. Trajectories of the jib head and the payload for control system simulation $\left(\varphi_{\text {end }}=1.57 \mathrm{rad}, \boldsymbol{l}=\mathbf{4 m}\right.$, wws $1=2$, wws $2=3$, wws $3=7$, inputs of controller: $\left.1 . \varphi_{\text {end }}-\varphi ; 2 . \dot{\boldsymbol{q}}\right)$ 
Moreover, Figs. 7-8 show the trajectories of a crane's motion for a situation in which the operator selects the target point before motion start. These tests were carried out to evaluate the effectiveness of fuzzy logic control.

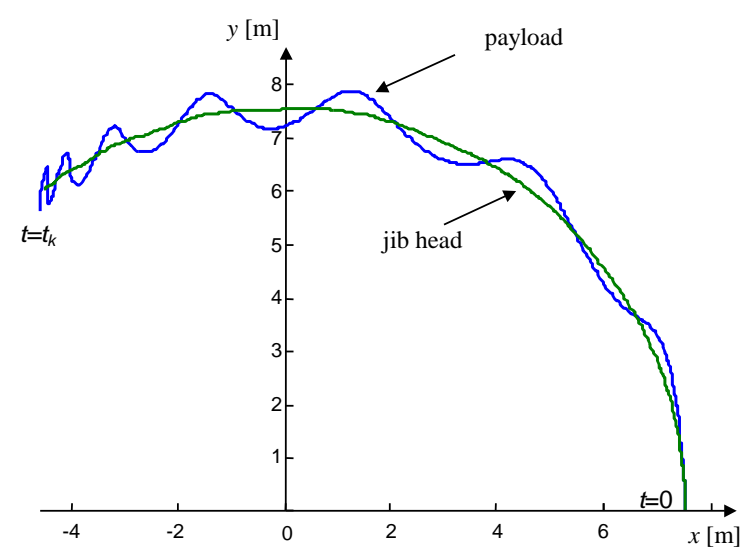

Fig. 8. Trajectories of the jib head and the payload for control system simulation $\varphi_{\text {end }}=\mathbf{2 r a d}, \boldsymbol{l}=\mathbf{4 m}$, wws $1=1$, wws2 $=2$, wws $3=1$, inputs of controller: 1. $\left.\varphi_{\text {end }}-\varphi ; \mathbf{2} . \boldsymbol{v}\right)$

\section{SUMMARY}

Control with the use of the FLC controller eliminates the need to formulate a control strategy before a motion starts and is less sensitive to the disturbances in the implementation of the formulated control strategy. However, such control does not always ensure considerable elimination of the oscillations of the payload remaining after a motion - this is particularly true of the radial component of oscillations, which is mainly generated by a centrifugal inertial force, i.e. a force that acts in a direction for which it is impossible to control motion by only using a rotation drive.

The carried out numerical simulations of the crane model will be used to select the suitable amplification coefficients of the controller as well as to propose the modification of control system in order to achieve to better acuracy of the load positioning after ending the motion.

\section{REFERENCES}

1. Al-Humaidi H. M., Hadipriono Tan F. (2009), Mobile crane safe operation approach to prevent electrocution using fuzzy-set logic models, Advances in Engineering Software, 40, 686-696.

2. Cho S. K., Lee H. H. (2002), A fuzzy-logic antiswing controller for three-dimensional overhead cranes, ISA Transactions, 41, 235-243.

3. Hong K. S., Ngo Q. H. (2012), Dynamics of the container crane on a mobile harbor, Ocean Engineering, 53, 16-24.

4. Janusz J., Kłosiński J. (2008), Fuzzy controlling of a mobile crane ensuring stable work, Acta Mechanica Slovaca, 3-C, 215-222.

5. Jerman B., Podrzaj P., Kramar J. (2004), An investigation of slewing-crane dynamics during slewing motion - development and verification of a mathematical model, International Journal of Mechanical Sciences, 46, 729-750.

6. Ju F., Choo Y. S. Cui F. S. (2006), Dynamic response of tower crane induced by pendulum motion of the payload, International Journal of Solids and Structures, 43, 376-389.

7. Kłosiński J. (2005), Swing-free stop control of the slewing motion of the mobile crane, Control Engineering Practice, 13, 451-460.
8. Kłosiński J. Majewski L. (2004), Numerical investigations of the system with fuzzy logic controller used to controlling the working motion of mobile crane, Proceedings of the IX Conference on the TMM, Liberec, 445-450.

9. Kłosiński J., Janusz J. (2009), Control of operational motions of a mobile crane under a threat of loss of stability, Solid State Phenomena, Vol.144, 77-82.

10. Kłosiński J. (2011), Fuzzy logic-based control of a mobile crane slewing motion, Mechanics and Mechanical Engineering, Vol. 15, No $4,73-80$.

11. Lee T. Y., Lee S. R. (2002), Anti-sway and position 3D control of the nonlinear crane system using fuzzy algorithm, Inter. Journal of the Korean Society of Precision Engineering, Vol.3, No.1, 66-75.

12. Liu D., Yi J., Zhao D., Wang W. (2005), Adaptive sliding mode fuzzy control for a two-dimensional overhead crane, Mechatronics, 15, 505-522.

13. Mahfouf M., Kee C. H., Abbod M. F., Linkens D. A. (2000), Fuzzy logic-based anti-sway control design for overhead cranes, Neural Computing \& Applications, 9, 38-43.

14. Neupert J., Arnold E., Schneider K., Sawodny O. (2010), Tracking and anti-sway control for boom cranes, Control Engineering Practice, 18, 31-44.

15. Pędrak T., Kłosiński J. (2009), Control of mobile crane by means of fuzzy logic controller, Solid State Phenomena, Vol.144, 202-207.

16. Schaub H. (2008), Rate-based ship-mounted crane payload pendulation control system, Control Engineering Practice, 16, 132-145.

17. Smoczek J. (2014), Fuzzy crane control with sensorless payload deflection feedback for vibration reduction, Mechanical Systems and Signal Processing, 46, 70-81.

18. Smoczek J., Szpytko J. (2012), Design of gain scheduling anti-sway controller using genetic fuzzy system, 17th IFAC Int. Conf. on Methods and Models in Automation and Robotics MMAR, 573-578.

19. Smoczek J., Szpytko J., Hyla, P. (2013), The anti-sway crane control system with using dynamic vision system, Solid State Phenomena, 198, 589-593.

20. Sochacki W. (2007), The dynamic stability of a laboratory model of a truck crane, Thin-Walled Structures, 45, 927-930.

21. Solihin M., Wahyudi I., Legowo A. (2010), Fuzzy-tuned antiswing control of automatic gantry crane, Journal of Vibration and Control, 16(1), 127-145.

22. Terashima K., Shen Y., Yano K. (2007), Modeling and optimal control of a rotary crane using the straight transfer transformation method, Control Engineering Practice, 15, 1179-1192.

23. Tomczyk J., Cink J., Kosucki A. (2014), Dynamics of an overhead crane under a wind disturbance condition, Automation in Construction, 42, 100-111.

24. Yi J., Yubazaki N., Hirota K. (2003), Anti-swing and positioning control of overhead traveling crane, Information Sciences, 155,19-42. 